\title{
Sleep disorders in pregnancy
}

Rosalia Silvestri ${ }^{1}$

Irene Aricò ${ }^{1}$

\footnotetext{
${ }^{1}$ University of Messina, Department of Clinical and Experimental Medicine, Sleep Medicine Centerof the Neurophysiopathology and Movement Disorders Unit - Messina - Messina Italy.
}

\begin{abstract}
Anatomical, physiological, psychological and hormonal alterations affect sleep during pregnancy. Sleep appears tobe commonly impaired only after the first trimester. Albeit objective data regarding the reduction of sleep durationand efficiency are not univocal, poor sleep is reported by over half of pregnant women. The reasons underlyingthese complaints are multiple, including lower back pain, gastroesophageal reflux disorder (GERD), increasedmicturition and repositioning difficulties at night. Specific primary sleep disorders whose prevalence drasticallyincreases during pregnancy include obstructive sleep apnea (OSA) and restless legs syndrome (RLS), both relatedto gestational hypertension and gestational diabetes mellitus (GDM). Pre-eclampsia and labor complicationsleading to an increased number of cesarean sections and preterm births correlate with insomnia and OSA inparticular. Post-partum depression (PPD) and impairment of the mother-infant relationship may also be consideredas secondary effects deriving from poor sleep during pregnancy. Recognition and treatment of sleep disordersshould be encouraged in order to protect maternal and fetal health and prevent dire consequences at birth.
\end{abstract}

Keywords: Pregnancy; Sleep Initiation and Maintenance Disorders; Sleep Apnea Syndromes; Restless Legs Syndrome; Diabetes, Gestational; Hypertension; Pregnancy-Induced.
Corresponding author: Rosalia Silvestri.

E-mail: rosalia.silvestri@unime.it Received: September 24, 2018; Accepted: June 5, 2019. 


\section{DIFFERENT FACTORS AFFECTING SLEEP IN PREGNANCY}

Changes in sleep during pregnancy reflect alterations of several modified body-mind aspects including anatomical, physiological, hormonal and psychological factors. Increased weight (up to $20 \%$ more than pre-gestational weight) and uterine volume comprise the most significant anatomical changes; uterine volume affects diaphragm elevation, leading to respiratory impairment. Body repositioning difficulties affect both sleep continuity and initiation. Primary physiological factors include cardio-respiratory changes such as increased pulse rate, blood pressure, and respiratory frequency, with an augmented alveolar/arterial oxygen gradient. Sympathetic activity, which is partially mediated by hormonal changes, is also increased during pregnancy, as are cardiac load and ejection fraction. All of these changes contribute to the fatigue and exhaustion endured by the expecting mother.

Pregnancy-related physiological changes include slower digestion due to the increase of gastric-emptying time, as well as constipation and gastroesophageal reflux. The latter, in fact, represent common problems in later pregnancy, affecting over $75 \%$ of pregnant women in some populations. Nocturnal micturition, which is linked to increased overnight sodium excretion, may also affect sleep fragmentation. ${ }^{1}$

Hormonal modifications are undoubtedly the most important factors affecting sleep length, quality, and physiology. Steroid hormones, namely estrogen and progesterone, increase during pregnancy with different and often complimentary effects on sleep and respiratory physiology. Progesterone's early rise during the first trimester enhances slow-wave sleep and activity due to the induction of GABA receptors ${ }^{2}$; it also acts as a respiratory drive stimulant, as in obese women, by increasing the activity of the genioglossus muscle, thus dilating the upper airway diameter. ${ }^{3}$

The counterpart of this protective effect against Obstructive Sleep Apnea (OSA) may be an increased risk for central apneas, due to hormonally-induced chemoreceptor resetting favoring hyperventilation/hypocapnia coupling, in addition to the increased pressure response to hypercapnia and apneas. ${ }^{4}$

Anxiety, stress and tension are powerful psychological mechanisms impacting sleep duration and quality, especially in primiparous women. A strong bidirectional relationship between sleep and mood has been established, with gestational insomnia strongly affecting the likelihood of post-partum depression (PPD). ${ }^{5}$

\section{INSOMNIA IN PREGNANCY}

Common complaints regarding the quality of sleep usually worsen during pregnancy, increasingly toward the end of the third trimester. ${ }^{4}$ The subjective quality of sleep is altered as early as in the first trimester, despite an increase in total sleep time. This deterioration of the subjective quality of sleep, along with the aforementioned progestinic effect, may be the underlying factors contributing to the frequently reported increase in excessive daytime sleepiness (EDS) during this time. Sleep fragmentation increases exponentially toward the end of pregnancy, ${ }^{6}$ with an important role played by snoring, respiratory effort-related arousals (RERAs), ${ }^{7}$ restless legs syndrome (RLS), ${ }^{8}$ and lower back pain and leg cramps, all affecting sleep efficiency and continuity.

Conversely, sleep reduction is less univocally reported by authors and only starts in the second trimester. In a wide survey of 486 young pregnant women', the likelihood of insomnia in pregnancy increased with age, low education levels, and BMI of 25 and over. However, logistic regression analysis did not confirm BMI as a risk factor. Subjects with depressive symptoms had a 2.6-fold increased risk of developing insomnia compared to non-depressed women. Comprehensively, more than half of the women reported insomnia symptoms despite normal sleep duration, at least during the first trimester, whereas a gradual decrease of total sleep time was observed as the pregnancy progressed.

Another study ${ }^{6}$ found that nearly $28 \%$ of pregnant women slept less than 7 hours per night during the second trimester. Both race and age had an impact on sleep duration, with nonHispanic, black, Asian and older women reporting the shortest sleep time. A recent meta-analysis of studies conducted through 2015 concerning sleep quality during pregnancy ${ }^{10}$ found that $46 \%$ of women reported poor sleep, with a mean Pittsburgh Sleep Quality Index (PSQI) of 6.4 (95\% CI: 5.3-6.85) and an increasing PSQI score (indicating worse sleep quality) from the second to the third trimester, by an average of 1.68 points $(95 \%$ CI: 0.42-2.94).

As in the general population, both poor sleep quality and short sleep duration are linked to adverse health consequences, including gestational hypertension. ${ }^{11}$ However, these results rely only on self-reported insomnia and were not confirmed in a low-risk population with objective sleep measures.

Cross-sectional studies using self-reported sleep measures including PSQI, and prospective longitudinal studies assessing sleep duration ( $<7$ hours) found an increased risk of gestational diabetes mellitus (GDM) to be independently associated to both short and poor sleep. BMI appears to be an important modifier of this relationship since both short and long sleep duration are associated with the risk of GDM, but only in non-obese women. ${ }^{12}$

Short sleep duration has also been reported to affect labor outcome and modalities, increasing both labor duration and the rate of cesarean deliveries. ${ }^{13}$

Given the well-known relationship between insomnia and depression, several studies aimed to explore the effect of poor sleep during pregnancy on PPD. Dørheim et al. ${ }^{14}$ found that insomnia did not predict PPD in women with a negative psychiatric history, whereas women who recovered from depression reported residual insomnia. On the other hand, high Edinburgh postnatal depression scale (EPDS) and anxiety scores during pregnancy, primiparity with fear of delivery, high educational levels, and prior depression were risk factors 
for both postpartum insomnia and depression. As for postpartum objective sleep measures, sleep duration (mean of 6.5 hours) and sleep efficiency reduction (from $84 \%$ to $75 \%$ ) were not paralleled by self-reported insomnia scores (Bergen Insomnia Scale). The latter, in fact, decreased from 17.2 to 15.4. In addition, the reported prevalence of insomnia symptoms also decreased from $61.6 \%$ during pregnancy to $53.8 \%$ post-partum. Comprehensively, women reported less daytime impairment and overall more sleep satisfaction post-partum compared to during pregnancy. Hence, insomnia by subjective assessment, rather than sleep efficiency or sleep duration during pregnancy, is a more accurate predictor of PPD, as previously shown by other studies. ${ }^{15}$

A recent randomized clinical trial was conducted on 54 pregnant women reporting insomnia during the third trimester; the effect of insomnia treatment versus placebo on PPD symptoms was evaluated. ${ }^{16}$ Women were divided into three groups and assigned to trazodone, diphenhydramine or placebo. Both objective sleep duration (actigraphy) and depressive symptoms (EPDS) were measured at baseline and at two and six weeks after delivery, showing equal improvement of sleep with drug treatment and a positive effect on depressive symptoms compared to placebo. Both trazodone and diphenhydramine are recognized as safe treatment options for gestational insomnia, hence their use may be advocated as a possible therapeutic preventative option, especially in the presence of depressive symptoms arising late during the gestational period.

A noteworthy paper ${ }^{17}$ explored the implications and harmful consequences of chronic sleep deprivation, REM reduction and changes in sleep-related hormone homeostasis on pregnant women. These changes lead to maternal fatigue, peri and post-natal depression and pediatric sleep problems, thereby potentially undermining the mother-infant relationship.

\section{GESTATIONAL SLEEP DISORDERED BREATH- ING}

Sleep disordered breathing (SDB) refers to breathing alterations during sleep, ranging from simple snoring to complete cessation of breathing (i.e. apneas) with airflow interruptions lasting at least 10 seconds, usually accompanied by oxygen desaturation and fragmented sleep. Hypopneas, instead, are defined as airflow interruptions $\geq 10$ seconds with $\geq$ a $50 \%$ reduction in airflow and a $3 \%$ desaturation. The severity of SDB is usually expressed in terms of apnea and hypopnea index (AHI; mild: 5 -15 events/hr; moderate: $15-30$ events/hr; severe: $\geq 30$ events/hr).

As previously explained in Section 1, progesterone has positive protective effects on upper airway dilation and respiratory drive. In fact, pregnant women with OSA show lower progesterone levels compared to controls. ${ }^{18}$ However, an increased diaphragmatic effort may also be due to a heightened respiratory drive, causing suction pressure at the level of the upper airways with secondary increased collapsibility. Functional residual capacity is also diminished by $20 \%$ toward the end of pregnancy due to diaphragm elevation, with consequentially reduced maternal oxygenation. Other risk factors for gestational SDB include estrogen-mediated nasopharyngeal edema and rhinitis, with increased airflow resistance and diminished upper airway patency, along with the notorious effect of increased AHI with weight gain. On the other hand, counter-acting gestational protective factors against SDB include maternal positional changes favoring a lateral positioning of the body, REM reduction, and the rightward shift of the oxyhemoglobin dissociation curve promoting placental oxygen delivery despite scanty maternal reserves. ${ }^{19}$

Snoring is drastically increased in pregnant women, mainly due to the estrogenic effect on the nasal mucosa. Loud snoring in the third trimester has an estimated prevalence between $14-45 \%$. RERAs, even in the absence of clear apneas, may be responsible for significant sleep fragmentation and increased sympathetic activation, leading to hypertension in the context of upper airway resistance syndrome (UARS). ${ }^{7}$

Habitual snoring was independently predictive of both hypertension (OR: $2.03 ; p<0.05)$ and small-for-gestational-age infants (OR: $3.45 ; p<0.001)$ in a logistic regression analysis controlling for weight, age, and smoking. ${ }^{20}$ Habitual snoring during early pregnancy also proved to be a risk factor for glucose intolerance, as well as for GDM.

Several studies have explored the prevalence of OSA in pregnancy. In normal low-risk pregnancies, the Nulliparous pregnancy outcomes study: monitoring mothers-to-be $(\mathrm{Nu}-$ MoM2b) ${ }^{21}$ found an OSA prevalence of $3.6 \%$ in early versus $8.3 \%$ in mid-pregnancy. However, BMI accounted as an important risk factor in this study, since pre-pregnancy BMI $>30$ was associated with a $10 \%$ prevalence of OSA in early pregnancy. Another study ${ }^{22}$ indicated that BMI and maternal age are statistically significant predictors of gestational OSA in a non-risk population, accounting for an increase of OSA prevalence from $10.5 \%$ in the first trimester to $26.7 \%$ in the third trimester.

In a high-risk pregnancy population with high BMI and one or more comorbid risk factors among chronic hypertension, pre-GDM, prior pre-eclampsia, or twin gestation, $30 \%$ of women in early, and $47 \%$ in late pregnancy met criteria for OSA $(\mathrm{AHI}>5 / \mathrm{h}) .^{23}$

No large-scale prospective data concerning the persistence of OSA post-pregnancy exists thus far. In a small cohort of patients, improvement without complete resolution of apneas was found three months post-partum. ${ }^{24}$

Several screening tools have been applied for the diagnosis of OSA in pregnancy, ranging from questionnaires such as the STOP-Bang Questionnaire (SBQ), the Berlin Questionnaire (BQ) and the Epworth Sleepiness Scale (ESS), to more accurate instrumental testing. Tantrakul et al. ${ }^{25}$ explored the performance and accuracy of the BQ and the ESS as screening tests with negative results, especially when they were performed during early pregnancy ( $<20$-weeks gestation), and high-risk pregnancies. The tests showed to be otherwise only moderately accurate in predicting gestational OSA, with a sensitivity between $35-73 \%$ and a specificity between 58-90\%. Subsequently, Facco et $a .^{26}$ developed a four-variable prediction rule using BMI, age, 
frequent snoring, and chronic hypertension to predict OSA in high-risk pregnancies, leading to an improved sensitivity (39$96 \%$ ) and specificity $(68-74 \%)$, compared to the use of the BQ alone.

A three-variable optimized model based on BMI $\geq 32$ $\mathrm{kg} \mathrm{m}^{-2}$, snoring volume and tiredness upon awakening has been proposed for women with uncomplicated pregnancies in the second trimester. Said model presents a consistently increased predictive performance of SDB with respect to the $\mathrm{BQ}$ and the Multivariate Apnea Risk (MAP) Index. ${ }^{27}$ In fact, stepwise logistic regression determined these three variables as the strongest independent predictors of OSA during pregnancy.

The importance of anatomical factors in the screening process for apneas led to the validation of a screening tool involving two variables, neck circumference and the $\mathrm{BQ}$, which proved to be critically important for the diagnostic algorithm of special populations at risk, such as women with GDM. ${ }^{28}$

An overnight attended polysomnogram (PSG) is still considered the gold standard test for the objective instrumental diagnosis of OSA, but is financially burdensome and can be challenging to perform during pregnancy. Home-ambulatory studies including at least four channels allow cardio-respiratory monitoring but, due to the absence of electroencephalogram parameters, they don't provide sleep scoring information (see Table 1).

Detrimental consequences of gestational OSA include maternal adverse health effects with gestational hypertension/ pre-eclampsia ${ }^{20,29}$ and gestational diabetes in early (OR: 3.47) and mid (OR: 2.79) pregnancy. Recently, a study diagnosed OSA in $52.4 \%$ of 82 obese pregnant women with diet-controlled GDM at a median gestational age of 29 weeks. ${ }^{28}$ More severe OSA was significantly correlated to higher fasting glucose but not $\mathrm{HbA1c}$, while oxygen desaturation correlated with insulin resistance and more severe $\beta$-cell dysfunction. A recently published meta-analysis ${ }^{30}$ assessing over 18,000 pregnancies found that extreme sleep duration during pregnancy was a risk factor for GDM. An updated systematic review and meta-analysis ${ }^{31}$ on maternal and fetal outcomes associated with gestational SDB reported an increased OR risk for GDM in pregnant snorers (OR: $2.14,95 \% \mathrm{CI}=1.73-2.81)$, more than in women later diagnosed with OSA (OR: 1.71, 95\% CI = 1.23-2.38). However, pregnant women with OSA presented a high risk of pre-term birth (OR $=1.75,95 \% \mathrm{CI}=1.21-2.55)$, whereas no significant risk was associated with pregnant snorers $(\mathrm{OR}=1.22,95 \% \mathrm{CI}=0.87$ 1.70). The authors assert that the unexpected greater influence of snoring, instead of OSA, on adverse maternal and fetal outcomes may be due to the inclusion and consideration of small cohort studies in the meta-analysis.

The activation of inflammatory-related cytokines and other serum markers, OSA-induced endovascular dysfunction related to hypoxia and oxidative damage could well account for pre-eclampsia mechanisms in pregnant women with SDB who also show alterations of placenta-secreted glycoproteins and markers of angiogenesis. The hypothalamic-pituitary axis may also play a role in the association of GDM with SDB. An increased inflammatory profile, endothelial and immune dysfunction and oxidative stress may represent additive factors.

Another hypothesis regards the allostatic load, suggesting that the detrimental effect of chronic sleep loss leads to a stress overload which, in turn, is responsible for adverse pregnancy outcomes.

Fetal consequences of SDB have been extensively documented in experimental animals, with long-term detrimental fetal effects and persistent metabolic dysfunction presenting in adulthood in the male, rather than female, offspring. ${ }^{32}$ Maternal snoring enhances fetal erythropoiesis with increased levels of nucleated red blood cells, erythropoietin, and interleukin-6 in the cord blood vessels. This effect may be mediated by both intermittent hypoxia and sympathetic overdrive on placental perfusion.

Labor and neonatal complications are also linked to gestational OSA and include increased labor duration and caesarian sections, stillbirths and pre-term births, infants with low birth weight (LBW), small for gestational age,,$^{20}$ and hypoxic brain

Table 1. Diagnostic Tests for Sleep Disordered Breathing in Pregnancy.

\begin{tabular}{l}
\hline Screening Questionnaires \\
\hline$\diamond$ Epworth Sleepiness Scale (ESS) \\
\hline$\diamond$ Berlin Questionnaire (BQ) \\
\hline$\diamond$ STOP-Bang Questionnaire (SBQ) \\
\hline Additional Screening Tools \\
\hline$\diamond$ BMI \\
\hline$\diamond$ Neck circumference \\
\hline$\diamond$ Mallampati Score \\
\hline$\diamond$ Snoring volume \\
\hline$\diamond$ Facco: 4 variable prediction rule \\
\hline$\diamond$ Algorithm for GDM associated to OSA in pregnancy \\
\hline Objective Instrumental Evaluation \\
\hline$\diamond$ Gold standard: attended video PSG \\
\hline$\diamond$ Home based ambulatory cardio-respiratory monitoring \\
\hline GDM: Gestational Diabetes Mellitus; OSA: Obstructive Sleep Apnea, PSG: Polysomnography.
\end{tabular}


damage with lower Apgar scores. A recent study revealed contrasting evidence, suggesting that fetal growth may actually be accelerated rather than stilted in non-obese pregnant women with mild maternal OSA due to abnormal adiposity distribution and placental perfusion. ${ }^{33}$ Most pre-term births are due to pre-eclampsia, suggesting that OSA represents an additive risk factor in high-risk pregnancies (see Table 2).

SDB treatment recommendations during pregnancy underscore the importance of sleep hygiene, including lateral positioning, adequate bed-timing, and avoidance of alcohol and other sedatives. On the contrary, oral appliances, surgical procedures, and supplemental night oxygen are not recommended during the gestational period (see Table 3).

No extensive reviews concerning the role of positive airway pressure ventilatory therapy during pregnancy exist to date. Edwards ${ }^{34}$ was among the first to recommend continuous positive airway pressure (CPAP) therapy to reduce nocturnal blood pressure in pre-eclampsia. Later studies conducted by Guilminault et al. ${ }^{35}$ confirmed a superior control of blood pressure with CPAP in women suffering from chronic hypertension and OSA. Recently, a single case report with a reversal of early pre-eclampsia by CPAP in a woman with severe apneas was published. ${ }^{36}$ An improved cardiac load in pre-eclampsia without adverse events had been previously reported, along with a positive effect on fetal growth during the third trimester. CPAP treatment is recommended for SDB with AHI $\geq 15$ (level A) in the general population. However, several authors broaden this recommendation to include hypertensive pregnant women with clinical symptoms and an AHI $>5$, despite a

Table 2. Detrimental Consequences of Sleep Disordered Breathing in Pregnancy: maternal and fetal consequences.

\begin{tabular}{lc}
\hline Maternal Effects & Fetal Consequences \\
\hline Maternal fatigue & Intrauterine growth retardation \\
\hline Gestational hypertension & Small for gestational age \\
\hline Gestational diabetes & Preterm birth \\
\hline Pre-eclampsia & Still births \\
\hline Placental abruption & Hypoxic brain damage \\
\hline C-section & Shorter telomer length \\
\hline
\end{tabular}

prospective longitudinal study reporting that nasal CPAP was insufficient to prevent negative pregnancy outcomes. ${ }^{35} \mathrm{~A}$ positive effect of CPAP on insulin secretion in GDM has also been reported, along with improved pregnancy outcomes. ${ }^{37}$ Thus far, however, no systematic studies have tested short and long term effects of non-invasive nocturnal ventilation on gestational OSA.

\section{RESTLESS LEGS SYNDROME}

Restless Legs Syndrome (RLS), otherwise referred to as Willis-Ekbom Disease as a tribute to the earliest authors describing the condition, was found to be associated with gestation by Ekbom himself. In fact, he performed the first known epidemiologic study on this association, reporting a prevalence of $11.3 \%$ of RLS among 486 pregnant women. Several studies followed before the diagnostic criteria were well-established, describing a prevalence between $12-27 \%$, later confirmed by multiple international studies that assessed a final prevalence of $26-30 \%$ of gestational RLS. ${ }^{8}$

Upon reviewing the original 1995 criteria established by the International RLS Study Group, the 2002 NIH epidemiologic workshop $^{38}$ provided the following diagnostic criteria, confirmed by the latest International Classification of Sleep Disorders (2014): an urge to move the legs, usually but not necessarily accompanied by uncomfortable and unpleasant sensations that begin or worsen during periods of rest or inactivity such as sitting or lying down, partially or totally relieved by movement such as walking or stretching, as long as the activity continues. These symptoms occur or worsen in the evening or night and should not solely be accounted for as primary to another medical or behavioral condition such as myalgia, leg cramps, positional discomfort, venous insufficiency or the likes. RLS symptoms cause concern, distress, sleep disturbance, and/or impairment in mental, physical, social, occupational, educational, and behavioral functioning.

The prevalence of RLS in women is known to be twice that in men. ${ }^{8}$ Steroid hormones, in particular, estradiol and serum ferritin which, after puberty, is consistently less in females than in males throughout their reproductive life, may account for this gender preference.

Table 3. Treatment recommendations for gestational OSA.

CPAP

$\diamond$ Recommended in SDB general population with AHI $\geq 15$ (level A)

$\diamond$ Recommended in hypertensive pregnant women with clinical symptoms and AHI $\geq 5$

$\diamond$ Improved cardiac output in pre-eclampsia without adverse effects

$\diamond$ However, early nasal CPAP was not sufficient to prevent pregnancy negative outcome in a prospective longitudinal study ${ }^{34}$

Sleep Hygiene: recommendations for pregnant women with snoring and mild OSA

$\diamond$ Lateral positioning

$\diamond$ Avoidance of alcohol and sedatives

$\diamond$ Bed timing

Not supported during pregnancy

$\diamond$ Oral appliances

$\diamond$ Surgical procedures

$\diamond$ Supplemental night oxygen

Sleep Sci. 2019;12(3):232-239 
The pathophysiology of RLS depends primarily on three interrelated factors: dysfunction of the nigrostriatal dopaminergic system, depletion of brain and serum ferritin, and genetic influences. Iron is the cofactor of tyrosine-hydroxylase, a limiting enzyme that enables the transformation of tyrosine into dopamine. Furthermore, iron metabolic pathways are often altered in genetic models of RLS.

Only a minority of women with RLS during pregnancy (6.5-9.9\%) were already affected by the syndrome prior to their pregnancy. ${ }^{8}$ Their symptoms usually worsened during the gestational period, with increasing prevalence and severity from the first $(15.6 \%)$ to the last $(38.8 \%)$ trimester, mildly decreasing during the last month of pregnancy. ${ }^{39}$ Several longitudinal studies have demonstrated the disappearance of symptoms at the time of delivery, maintained thereafter throughout the puerperium. However, some authors found a high $(34.8 \%)$ percentage of women were still symptomatic after delivery. ${ }^{39}$ Residual postpartum RLS correlated with lower ferritin, multiparity and symptom onset during the second trimester.

Predictive risk factors for gestational RLS include, besides low ferritin, a positive family history, age, multiparity with no aggravating effect after three pregnancies, ${ }^{8}$ EDS, nocturnal leg cramps and snoring. There is, in fact, an important relationship between OSA and RLS that has recently received considerable attention. Proposed mechanisms for this relationship regard local tissue recurrent hypoxia; CPAP treatment for OSA demonstrated a favorable effect on RLS morbidity. ${ }^{40}$

Even if the immediate prognosis for gestational RLS is propitious in the short-term, the long-term one appears less favorable. In fact, in a follow-up study, Cesnick et al..$^{41}$ demonstrated a four-fold increased risk of developing chronic RLS in women who presented symptoms in a previous pregnancy, with an incidence of $56 \%$ person/year against $12.6 \%$ person/year in women who had not previously experienced RLS.

Age and pregnancy both act as important precipitants of symptoms in female RLS. Interestingly, the prevalence of RLS after age 60 is similar in both genders and nulliparous women present the same RLS prevalence of men.

The pathogenesis of RLS onset during pregnancy is still unclear. Both family history of RLS and multiparity have been recognized as independent predictors. Considering that not all women develop novel transient RLS symptoms during pregnancy, but all women who were already symptomatic pregestationally experience symptom exacerbation, one could speculate that both individual predisposition and specific pregnancyrelated factors play a role. Among the latter, factors related to iron storage and folate have received controversial coverage, owing to some authors reporting a protective effect from early supplementation that was not confirmed by other studies. ${ }^{8}$ The rise of steroid hormones during pregnancy has also been proposed as a possible mechanism accounting for the onset of new symptoms. An early rise of estradiol during the first trimester has been observed in pregnant women with RLS. ${ }^{42}$ Nonetheless, these results have not been confirmed by subsequent studies. Both estradiol and progesterone increase during pregnancy, peaking at the third trimester, and interact with dopamine in the striatum. Data from animal studies show that prolonged exposure to high concentrations of $17 \beta$-estradiol in the striatal tissue of female rats reduces striatal dopamine responsiveness. ${ }^{43}$

Increased levels of thyroid hormones during pregnancy may also contribute to the onset of symptoms, given the negative relationship between the latter and dopamine. ${ }^{8}$

Gestational RLS is aggravated by several comorbidities that may endanger pregnancy outcomes and maternal wellbeing; among them: mid-pregnancy and PPD, ${ }^{4}$ GDM (OR: 3.7), and gestational-hypertension with preeclampsia (OR: 2.1). An inverse relationship between the severity of symptoms and mother's weight and age at delivery has also been reported, ${ }^{8}$ but none of these studies were controlled for OSA, which often appears to be comorbid with RLS, as previously mentioned.

Poor quality of sleep and EDS were reported in an extensive cohort of pregnant women with RLS, ${ }^{44}$ with and without adverse delivery outcomes.

Consensus clinical practice guidelines for the treatment of RLS during pregnancy and lactation ${ }^{45}$ indicate the importance of considering non-pharmacological treatments and simple reassurance whenever symptom severity allows it. Moderate-intensity exercise, yoga, massages, pneumatic compression devices and treatment of OSA should always be attempted before initiating any type of pharmacological therapy. The latter should favor folate and oral iron supplementation for ferritin $<$ $75 \mathrm{mcg} / \mathrm{L}$ or IV iron sucrose for ferritin $<30 \mathrm{mcg} / \mathrm{L}$. Low dose clonazepam may be considered for the treatment of refractory RLS during the second and third trimester of pregnancy and lactation (0.25-1 $\mathrm{mg}$ in the evening), but concurrent use of diphenhydramine or anticonvulsants should be avoided.

As for first-line drugs commonly used in the general treatment of RLS, among $\alpha 2 \delta$ ligands, gabapentin (GBP; 300$900 \mathrm{mg}$ in the evening) is recommended for refractory symptoms, whereas there is insufficient data supporting the use of GBP enacarbil and pregabalin during pregnancy.

There is no evidence of major malformations or other detrimental outcomes from daily use of 50-200 $\mathrm{mg}$ of carbido$\mathrm{pa} /$ levodopa to reduce the risk of augmentation; however, the latter are not to be used in combination with benserazide, due to possible adverse effects on bone development. Dopaminergic drugs also inhibit lactation via prolactin suppression, but this is not a long-term effect when they are used during pregnancy. Not enough data is yet available to support the safe use of dopamine agonists such as pramipexole, ropinirole or rotigotine during gestation (See Table 4).

Opiates are highly effective for RLS treatment, but there is greater peril of congenital heart disease in opiate-exposed infants, in addition to the risk of neonatal abstinence/withdrawal syndrome. Bupropion, a dopaminergic antidepressant, rather than SSRIs, is recommended for the treatment of depression comorbid to RLS. The latter should be used starting from the second trimester in order to prevent possible related adverse outcomes including LBW, pre-term delivery, pre-eclampsia, and substance abuse. 
Table 4. Restless Legs Syndrome in Pregnancy: epidemiology, risks and management.

$\diamond$ Prevalence around $25-30 \%$, peaking in the third trimester, increasing with maternal age and parity

$\diamond$ Increased chance of developing permanent RLS after several affected pregnancies

$\diamond$ The role of steroid hormones is controversial

$\diamond$ Iron and folate supplementation abate prevalence and should be indicated as first-line options

$\diamond$ Non-pharmacologic treatment (CBT, exercise, massage, yoga) always advised as safest treatment options

$\diamond$ Clonazepam, gabapentin and carbi/levodopa without benserazide: best drug options for safety concerns, for severe RLS on or after second trimester CBT: Cognitive Behavioral Therapy; RLS: Restless Legs Syndrome.

\section{FINAL CONSIDERATIONS}

Sleep plays an essential role in the wellbeing of pregnant mothers and their infants. Deficient sleep may negatively affect maternal health and fetal outcome at birth, therefore all possible efforts should be encouraged to heed pregnant women's complaints regarding unrestful sleep and EDS. An adequate and thorough screening for major sleep disorders, in particular, OSA and RLS, should be mandatory in the obstetric practice. Psychological counseling and psychiatric evaluation should also be offered to primiparous and pregnant women with a personal history of anxiety and depressive disorders.

An attentive and timely therapeutic approach reflecting the multifaceted interactions of anatomical, physiological, psychological and hormonal alterations experienced by pregnant women is indispensable to prevent avertible maternal morbidity and adverse birth outcomes.

\section{REFERENCES}

1. Lee KA. Alterations in sleep during pregnancy and postpartum: a review of 30 years of research. Sleep Med Rev. 1998; 2:231-42.

2. Lancel M, Faulhaber J, Holsboer F, Rupprecht R. Progesterone induces changes in sleep comparable to those of agonistic GABAA receptor modulators. Am J Phys-Endocrinol Met. 1996; 271. doi:10.1152/ ajpendo.1996.271.4.e763.

3. Popovic RM, White DP. Upper airway muscle activity in normal women: influence of hormonal status. J Appl Physiol. 1998; 84:1055-62.

4. Bazalakova M. Sleep Disorders in Pregnancy. Semin Neurol. 2017; 37(6):661-8.

5. Okun ML, Hanusa BH, Hall M, Wisner KL. Sleep complaints in late pregnancy and the recurrence of postpartum depression. Behav Sleep Med. 2009; 7(2):106-17.

6. Reid KJ, Facco FL, Grobman WA, Parker CB, Herbas M, Hunter S, et al. Sleep during pregnancy: The nuMoM2b pregnancy and sleep duration and continuity study. Sleep. 2017; 40(5).

7. Truong KK, Guilleminault C. Sleep disordered breathing in pregnant women: maternal and fetal risk, treatment considerations, and future perspectives. Expt Rev Resp Med. 2018; 12:177-89.

8. Manconi M, Ulfberg J, Berger K, Ghorayeb I, Wesström J, Fulda S, et al. When gender matters: Restless legs syndrome. Report of the "RLS and woman" workshop endorsed by the European RLS Study Group. Sleep Med Rev. 2012; 16:297-307.

9. Kizilirmak A, Timur S, Kartal B. Insomnia in pregnancy and factors related to insomnia. Sci World J; 2012.

10. Sedov ID, Cameron EE, Madigan S, Tomfohr-Madsen LM. Sleep quality during pregnancy: a meta-analysis. Sleep Med Rev. 2018; 38:168-76.

11. Williams MA, Miller RS, Qiu C, Cripe SM, Gelaye B, Enquobahrie D. Associations of early pregnancy sleep duration with trimester-specific blood pressures and hypertensive disorders in pregnancy. Sleep. 2010; 33(10):1363-71

12. Facco FL, Grobman WA, Reid KJ, Parker CB, Hunter SM, Silver RM, et al. Objectively measured short sleep duration and later sleep midpoint in pregnancy are associated with a higher risk of gestational diabetes. Am J Obstet Gynecol. 2017; 217(4):447.e1-447.e13.

13. Plancoulaine S, Flori S, Bat-Pitault F, Patural H, Lin JS, Franco P. Sleep trajectories among pregnant women and the impact on outcomes: a population-based cohort study. Matern Child Health J. 2017; 21(5):1139-46.
14. Dørheim SK, Bjorvatn B, Eberhard-Gran M. Can insomnia in pregnancy predict postpartum depression? A longitudinal, population-based study. PLoS One. 2014; 9(4).

15. Park EM, Meltzer-Brody S, Stickgold R. Poor sleep maintenance and subjective sleep quality are associated with postpartum maternal depression symptom severity. Arch Womens Ment Health. 2013; 16(6):539-47.

16. Khazaie H, Ghadami MR, Knight DC, Emamian F, Tahmasian M. Insomnia treatment in the third trimester of pregnancy reduces postpartum depression symptoms: a randomized clinical trial. Psychiatry Res. 2013; 210(3):901-5.

17. Pires GN, Andersen ML, Giovenardi M, Tufik S. Sleep impairment during pregnancy: possible implications on mother-infant relationship. Med Hypotheses. 2010; 75(6):578-82.

18. Lee J, Eklund EE, Lambert-Messerlian G, Palomaki GE, Butterfield $\mathrm{K}$, Curran P, et al. Serum progesterone levels in pregnant women with obstructive sleep apnea: a case control study. J Women's Health. 2017; 26(3):259-65.

19. Silvestri R, Aricò I. Sleep-disordered breathing in pregnancy. Cur Resp Care Rep. 2013; 2:118-22.

20. Franklin KA, Holmgren PÅ, Jönsson F, Poromaa N, Stenlund H, Svanborg E. Snoring, pregnancy-induced hypertension, and growth retardation of the fetus. Chest. 2000; 117(1):137-41.

21. Haas DM, Parker CB, Wing DA, Parry S, Grobman WA, Mercer BM, et al. A description of the methods of the Nulliparous Pregnancy Outcomes Study: Monitoring mothers-to-be (nuMoM2b). Am J Obstet Gynecol. 2015; 212(4):539.e1-539.e24.

22. Pien GW, Pack AI, Jackson N, Maislin G, Macones GA, Schwab RJ. Risk factors for sleep-disordered breathing in pregnancy. Thorax. 2014; 69(4):371-7.

23. Facco FL, Ouyang DW, Zee PC, Grobman WA. Sleep disordered breathing in a high-risk cohort prevalence and severity across pregnancy. Am J Perinatol. 2014; 31(10):899-904.

24. Edwards N, Blyton DM, Hennessy A, Sullivan CE. Severity of sleep-disordered breathing improves following parturition. Sleep. 2005; 28(6):73741.

25. Comer DK, Clark CR, Canelas DA. Writing to learn and learning to write across the disciplines: Peer-to-peer writing in introductory-level MOOCs. Int Rev Res Open Distance Learn. 2014; 15(5):26-82.

26. Facco FL, Ouyang DW, Zee PC, Grobman WA. Development of a pregnancy-specific screening tool for sleep apnea. J Clin Sleep Med. 2012; 8(4):389-94.

27. Wilson DL, Walker SP, Fung AM, O'Donoghue F, Barnes M, Howard $\mathrm{M}$. Can we predict sleep-disordered breathing in pregnancy? The clinical utility of symptoms. J Sleep Res. 2013; 22(6):670-8.

28. Wanitcharoenkul E, Chirakalwasan N, Amnakkittikul S, Charoensri S, Saetung S, Chanprasertyothin S, et al. Obstructive sleep apnea and dietcontrolled gestational diabetes. Sleep Med. 2017; 39:101-7.

29. O'Brien LM, Bullough AS, Chames MC, Shelgikar A V., Armitage R, Guilleminualt $C$, et al. Hypertension, snoring, and obstructive sleep apnoea during pregnancy: A cohort study. BJOG An Int J Obstet Gynaecol. 2014; 121(13):1685-93.

30. Xu YH, Shi L, Bao YP, Chen SJ, Shi J, Zhang RL, et al. Association between sleep duration during pregnancy and gestational diabetes mellitus: a meta-analysis. Sleep Med. 2018; 52:67-74.

31. Li L, Zhao K, Hua J, Li S. Association between sleep-disordered breathing during pregnancy and maternal and fetal outcomes: An updated systematic review and meta-analysis. Vol. 9. Front Neurol; 2018.

32. Khalyfa A, Cortese R, Qiao Z, Ye H, Bao R, Andrade J, et al. Late gestational intermittent hypoxia induces metabolic and epigenetic changes in male adult offspring mice. J Physiol. 2017; 595(8):2551-68.

33. Telerant A, Dunietz GL, Many A, Tauman R. Mild maternal obstructive sleep apnea in non-obese pregnant women and accelerated fetal growth. Sci Rep. 2018; 8(1). 
34. Edwards N, Blyton DM, Kirjavainen T, Kesby GJ, Sullivan CE. Nasal continuous positive airway pressure reduces sleep-induced blood pressure increments in preeclampsia. Am J Respir Crit Care Med. 2000; 162(1):252-7.

35. Guilleminault C, Palombini L, Poyares D, Takaoka S, Huynh NTL, ElSayed Y. Pre-eclampsia and nasal CPAP: Part 1. Early intervention with nasal CPAP in pregnant women with risk-factors for pre-eclampsia: Preliminary findings. Sleep Med. 2007; 9(1):9-14.

36. Whitehead C, Tong S, Wilson D, Howard M, Walker SP. Treatment of early-onset preeclampsia with continuous positive airway pressure. $\mathrm{Ob}$ stet Gynecol. 2015; 125(5):1106-9.

37. Chirakalwasan N, Amnakkittikul S, Wanitcharoenkul E, Charoensri S, Saetung S, Chanprasertyothin S, et al. Continuous positive airway pressure therapy in gestational diabetes with obstructive sleep apnea: A randomized controlled trial. J Clin Sleep Med. 2018; 14(3):327-36.

38. Allen RP, Picchietti D, Hening WA, Trenkwalder C, Walters AS, Montplaisi J, et al. Restless legs syndrome: Diagnostic criteria, special considerations, and epidemiology. A report from the restless legs syndrome diagnosis and epidemiology workshop at the National Institutes of Health. Sleep Med. 2003; 4(2):101-19.

39. Neyal A, Senel GB, Aslan R, Nalbantoglu M, Acikgoz S, Yilmaz N, et al. A prospective study of Willis-Ekbom disease/restless legs syndrome during and after pregnancy. Sleep Med. 2015; 16(9):1036-40.
40. Rodrigues RND, Rodrigues AAA de AS, Pratesi R, Krieger J. Outcome of restless legs severity after continuous positive air pressure (CPAP) treatment in patients affected by the association of RLS and obstructive sleep apneas. Sleep Med. 2006; 7(3):235-9.

41. Cesnik E, Casetta I, Turri M, Govoni V, Granieri E, Ferini Strambi L, et al Transient RLS during pregnancy is a risk factor for the chronic idiopathic form. Neurology. 2010; 75(23):2117-20.

42. Dzaja A, Wehrle R, Lancel M, Pollmächer T. Elevated estradiol plasma levels in women with restless legs during pregnancy. Sleep. 2009; 32(2):169-74.

43. Becker JB. Direct effect of 17beta-estradiol on striatum: Sex differences in dopamine release. Synapse. 1990; 5(2):157-64.

44. Dunietz GL, Lisabeth LD, Shedden K, Shamim-Uzzaman QA, Bullough AS, Chames MC, et al. Restless legs syndrome and sleep-wake disturbances in pregnancy. J Clin Sleep Med. 2017;13(7):863-70.

45. Picchietti DL, Hensley JG, Bainbridge JL, Lee KA, Manconi M, McGregor JA, et al. Consensus clinical practice guidelines for the diagnosis and treatment of restless legs syndrome/Willis-Ekbom disease during pregnancy and lactation. Sleep Med Rev. 2015; 22:64-77. 